\title{
Long-Term Impact of COVID-19: A Systematic Review of the Literature and Meta-Analysis
}

\author{
Diana C. Sanchez-Ramirez ${ }^{1, * \mathbb{D}}$, Kaylene Normand ${ }^{1}$, Zhaoyun Yang ${ }^{2} \mathbb{D}$ and Rodrigo Torres-Castro ${ }^{3,+}$ \\ 1 Department of Respiratory Therapy, College of Rehabilitation Sciences, University of Manitoba, \\ Winnipeg, MB R3E 0T6, Canada; normandk@myumanitoba.ca \\ 2 School of Nursing, Jilin University, Changchun 130012, China; yzy13553100125@163.com \\ 3 Department of Physical Therapy, Faculty of Medicine, University of Chile, Santiago 8320000, Chile; \\ rodritorres@uchile.cl \\ * Correspondence: diana.sanchez-ramirez@umanitoba.ca \\ + International Physiotherapy Research Network (PhysioEvidence).
}

Citation: Sanchez-Ramirez, D.C.; Normand, K.; Yang, Z.; Torres-Castro, R. Long-Term Impact of COVID-19: A Systematic Review of the Literature and Meta-Analysis. Biomedicines 2021, 9, 900. https://doi.org/10.3390/ biomedicines 9080900

Academic Editors: Mauro Maniscalco and Pasquale Ambrosino

Received: 7 July 2021

Accepted: 23 July 2021

Published: 27 July 2021

Publisher's Note: MDPI stays neutral with regard to jurisdictional claims in published maps and institutional affiliations.

Copyright: (c) 2021 by the authors. Licensee MDPI, Basel, Switzerland. This article is an open access article distributed under the terms and conditions of the Creative Commons Attribution (CC BY) license (https:// creativecommons.org/licenses/by/ $4.0 /)$.

\begin{abstract}
Background: The long-term impact of COVID-19 is still unknown. This study aimed to explore post COVID-19 effects on patients chest computed tomography (CT), lung function, respiratory symptoms, fatigue, functional capacity, health-related quality of life (HRQoL), and the ability to return to work beyond 3 months post infection. Methods: A systematic search was performed on PubMed, Web of Science, and Ovid MEDLINE on 22 May 2021, to identify studies that reported persistent effects of COVID-19 beyond 3 months follow-up. Data on the proportion of patients who had the outcome were collected and analyzed using a one-group meta-analysis. Results: Data were extracted from 24 articles that presented information on a total of 5323 adults, postinfection, between 3 to 6 months after symptom onset or hospital discharge. The pooled prevalence of CT abnormalities was 59\% (95\% CI 44-73, $\mathrm{I}^{2}=96 \%$ ), abnormal lung function was 39\% (95\% CI 24-55, $\left.\mathrm{I}^{2}=94 \%\right)$, fatigue was $38 \%\left(95 \%\right.$ CI $\left.27-49, \mathrm{I}^{2}=98 \%\right)$, dyspnea was $32 \%\left(95 \%\right.$ CI $\left.24-40, \mathrm{I}^{2}=98 \%\right)$, chest paint/tightness was $16 \%\left(95 \%\right.$ CI $\left.12-21, \mathrm{I}^{2}=94 \%\right)$, and cough was $13 \%$, (95\% CI 9-17, $\left.\mathrm{I}^{2}=94 \%\right)$. Decreased functional capacity and HRQoL were found in 36\% (95\% CI 22-49, $\mathrm{I}^{2}=97 \%$ ) and 52\% (95\% CI 33-71, $\mathrm{I}^{2}=94 \%$ ), respectively. On average, 8 out of 10 of the patients had returned to work or reported no work impairment. Conclusion: Post-COVID-19 patients may experience persistent respiratory symptoms, fatigue, decreased functional capacity and decreased quality of life up to 6 months after infection. Further studies are needed to establish the extent to which post-COVID-19 effects continue beyond 6 months, how they interact with each other, and to clarify their causes and their effective management.
\end{abstract}

Keywords: COVID-19; persistent symptoms; long-term effects; follow-up; lung function; respiratory symptoms; fatigue; functional capacity; quality of life

\section{Background}

The novel coronavirus disease 2019 (COVID-19), caused by severe acute respiratory syndrome coronavirus 2 (SARS-CoV-2) infection, has spread rapidly worldwide reaching over 191 million confirmed cases, including 4.1 million deaths as of 21 July 2021 [1]. Although lung abnormalities are found even in asymptomatic patients with COVID-19 [2], most of the cases $(81 \%)$ have no or mild pneumonia and can be managed at home. Severe $(14 \%)$ and critical (5\%) cases develop severe pneumonia and respiratory failure, which require in-hospital oxygen treatment or mechanical ventilation and are more likely to have long-term effects [3].

Evidence from literature on viral pneumonia indicates that many recovered patients have significant lung changes and are affected by acute respiratory distress syndrome (ARDS), which can negatively impact their lung, physical function and quality of life for months or years [4,5]. Previous studies conducted on patients with severe acute respiratory 
syndrome (SARS) caused by a SARS coronavirus that was identified in 2003 report longterm decreases in pulmonary function, including reduced lung volume measurement in $7.3 \%$ of the patients at 6 months [6]. The most common pulmonary function impairment identified at a 1 year time point was of the forced expiratory volume (FEV1) and diffusing lung capacity for carbon monoxide (DLCO) which were still abnormal in $18.2 \%$ and $52.7 \%$ of patients, respectively, at 24 months post-SARS [7,8]. Decreases in exercise capacity and health-related quality of life were also found in SARS patients, and only $78 \%$ of SARS survivors had returned to work within 24 months [7]. Similarly, signs of severe abnormal pulmonary function were reported in $54.2 \%$ of patients with influenza A (H1N1), 1 year post-discharge [9], 59\% of the patients reported significant exertion dyspnea, 70\% had returned to work, health-related quality of life was poorer than for sex- and agematched general population groups [10]. Overall, evidence indicates that ARDS patients experience decreases in lung function, exercise endurance, levels of body function (muscle strength, walking capacity, and/or physical activity), and HRQoL, and lower return-towork rate [11-13]. As COVID-19 has recently emerged, the long-term impact of the disease, defined as the signs and symptoms lasting beyond the period of active infection, is just becoming known.

In recent months, the scientific literature has used the term "Long COVID" to describe illness in people who have reported lasting effects of the infection or have had the usual symptoms for far longer than would be expected [14]. Abnormal chest computed tomography $(\mathrm{CT})$ findings and impairment in lung function were found in COVID-19 patients 30 days after hospital discharge [15]. Persistent symptoms such as cough, dyspnea, and fatigue have commonly been reported in post COVID-19 patients between 30 and 56 days after hospital discharge [15-19]. Decreased functional capacity [17] and difficulties with the activities of daily living such as mobility and self-care [17-19] were also identified in patients for follow-ups of up to 8 weeks [20]. A study reports that $39 \%$ of patients perceived a decrease in their overall health [19] around 6 weeks after symptom onset. $61.5 \%$ of patients delayed their return to work at least 5 weeks from symptom onset, the primary reason for which was fatigue and weakness [16], and 38\% were absent due to illness [19]. Evidence indicates that COVID-19 can have significant persistent effects on patient outcomes up to 12 weeks after the period of acute infection [21-23]. However, the long-term impact of COVID-19 on patient outcomes is still unknown. The aim of this study is to explore persistent effects of COVID-19 beyond 3 months follow-up on patient chest computed tomography (CT), lung function, respiratory symptoms, fatigue, functional capacity, quality of life, and the ability to return to work. The results of this study will help fill a significant knowledge gap about the lingering effects of COVID-19 on patient outcomes, which are valuable for tailoring rehabilitation programs to the needs of COVID-19 patients and for informing future health care planning and resource allocation.

\section{Methods}

\subsection{Protocol and Registration}

This systematic review of the literature was conducted using Preferred Reporting Items for Systematic Reviews and Meta-Analyses (PRISMA) [24]. It was registered in the International Prospective Register for Systematic Reviews (PROSPERO) (CRD42021256958).

\subsection{Literature Search and Study Selection}

A systematic review was performed in the electronic bibliographic databases of PubMed, Web of Science and Ovid MEDLINE on 22 May 2021. The key words and the search strategy used were: ("COVID-19" OR "COVID19" OR "SARS-2" OR "Severe acute respiratory syndrome 2") AND ("follow-up" OR "follow-up studies" OR "lingering symptoms" OR "persistent symptoms" OR "long-term symptoms"). The search was limited to full text articles published in English. In addition, the reference lists of the selected studies were checked to retrieve relevant publications that were not found with the computerized search. 
A total of 24 publications met the inclusion criteria of: (1) reporting the effect of COVID-19 on one or more of the following outcomes in adult patients' lung imaging, lung function, respiratory symptoms, fatigue, functional capacity, activity limitations, overall health, quality of life, or return to work; (2) the mean or median study follow-up was longer than 3 months; (3) the publication was not a meta-analysis or systematic review. The articles selected were appraised by two review authors (RT and YZ) using the tool recommended by the National Heart, Lung, and Blood Institute (NHLBI) for the quality assessment of observational cohort and cross-sectional studies [25].

The systematic search retrieved 5054 references and 13 references were identified from other sources such as automatic email alerts on COVID-related databases, reference lists of identified studies, links shared on social media, suggested by a researcher, etc. After removing duplicates, two researchers (DS and KN) screened 2390 titles and abstracts and read in full the text of 74 articles. Both researchers independently reviewed the articles and selected 24 publications that met the inclusion criteria (Figure 1). Primary reasons for exclusion of the studies included; (1) the study did not present the effect of COVID-19 on any of the following outcomes: lung imaging, lung function, respiratory symptoms, fatigue, functional capacity, activity limitations, overall health, quality of life, and/or the ability to return to work; (2) the study was completed in children or in a group of post COVID-19 adults with a specific characteristic or disease (e.g., among patients with diabetes, or kidney problems); (3) the follow-up was less than 3 months; and (4) the article did not present primary data (e.g., protocol, opinion, etc.).

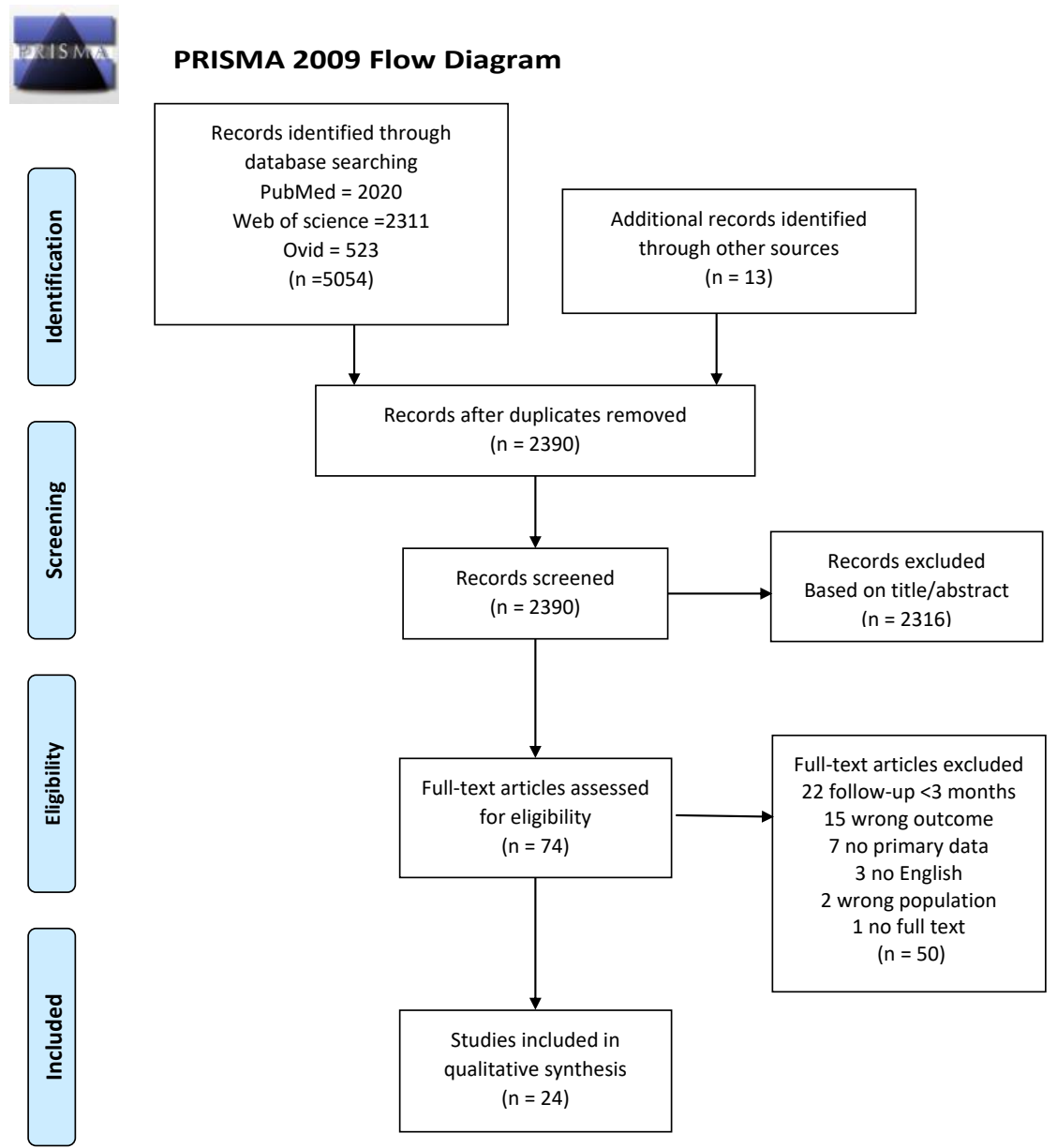

Figure 1. Diagram flow of studies screened and included in the review and meta-analysis. 


\subsection{Data Extraction and Synthesis}

Information from the 24 articles is synthesized in Table 1 which presents (1) author(s)' name, (2) country of the study, (3) study design, (4) follow-up time, (5) description of the population studied and, (6) disease severity at baseline. Prevalence of persistent COVID19 effects beyond 3 months of follow-up on chest CT scan, lung function, respiratory symptoms, fatigue, functional capacity, quality of life, and return to work of patients was extracted and is compiled in Table 2.

Review Manager (RevMan; version 5.3; Copenhagen, Denmark) software was used to pool the individually included studies. The analysis is presented as prevalence based on the presence of the outcome using one-group meta-analysis, and random-effects methods. To investigate heterogeneity between studies, the authors used the $\mathrm{I}^{2}$ index which describes the percentage of variation across the studies in the pooled analysis that is due to inconsistency rather than by chance. The $\mathrm{I}^{2}$ statistic was used to present between-study heterogeneity, where $\mathrm{I}^{2} \leq 30 \%$, between $30 \%$ and $50 \%$, between $50 \%$ and $75 \%$, and $\geq 75 \%$ were considered to indicate low, moderate, substantial, and considerable heterogeneity, respectively [26].

\section{Results}

This review identified a total of 24 articles that explore persistent effects of COVID-19 beyond 3 months of follow-up on patients lung imaging, lung function, respiratory symptoms, fatigue, functional capacity, HRQoL, and/or the ability to return to work [27-50]. The studies were completed in China (7), Canada (3), France (2), Norway (2), Italy (3), USA (2), Switzerland (1), Austria (1), Iran (1), the Netherlands (1), and the UK (1). Persistent outcomes were reported in 5323 post COVID-19 adult patients at an average of 4 (range 3-6) months of follow-up after symptom onset or hospital discharge. Mean age was $55.2 \pm 8.1$ years, and $56 \%$ of the patients studied were male. Severe illness due to COVID-19, defined as the presence of pneumonia, serious or critical illness, need for hospitalization, ICU care, use of supplemental oxygen, etc., was reported in approximately $36 \%$ of the cohort at baseline. Further description of the studies is presented in Table 1. Two of the Canadian studies $[35,37]$ presented information on the same population. Data from Wong et al. [35] were preferred when the same outcomes are reported in both studies due to longer follow-up and a larger number of patients studied; otherwise, the unique results presented in each study were included in this review.

The NHLBI' Quality Assessment Tool for Observational Cohort and Cross-Sectional Studies was used to evaluate the quality of the 24 studies included in the review. Nineteen studies were rated as "fair" and five rated as "poor" (Supplementary Table S1).

\subsection{Lung Imaging}

Thirteen articles report the results of chest CT scans in post COVID-19 patients at a mean of 3.8 (SD 1.3) months follow-up [28-34,36,37,40,41,43,45] (Supplementary Table S2 and Figure S1). Persistent chest CT abnormalities attributable to COVID-19 were identified in $59 \%$ of the patients ( $95 \%$ CI $44-73, \mathrm{I}^{2}=96 \%$ ) (Table 2). The most frequent $\mathrm{CT}$ abnormality was ground glass opacity (GGO) in $39 \%$ of the patients ( $95 \%$ CI $26-52, I^{2}=97 \%$ ), followed by interstitial thickening or interlobular septal thickening in 33\% (95\% CI 13-52, $\left.\mathrm{I}^{2}=98 \%\right)$, parenchymal band or fibrous stripe in 31\% (95\% CI 17-44, $\left.\mathrm{I}^{2}=95 \%\right)$, bronchovascular bundle distortion or bronchiectasis in $26 \%$ (95\% CI 9-43, $\left.\mathrm{I}^{2}=97 \%\right)$, thickening or adjacent pleura in $11 \%\left(95 \%\right.$ CI $\left.2-20, \mathrm{I}^{2}=94 \%\right)$, and consolidations in $6 \%\left(95 \%\right.$ CI $\left.2-11, \mathrm{I}^{2}=89 \%\right)$. One study reports crazy pavement in $5 \%$ of participants a mean of 93 days after discharge from hospital. Residual lung lesions at follow-up were associated with disease severity during the infectious period in some studies $[30,33,34,41,45]$ (Supplementary Table S2 and Figure S1). 
Table 1. Characteristics of the studies included $(n=24)$.

\begin{tabular}{|c|c|c|c|c|c|c|c|}
\hline \multirow{2}{*}{$\begin{array}{l}\text { Author } \\
\text { Year }\end{array}$} & \multirow{2}{*}{ Country } & \multirow{2}{*}{ Study Design } & \multirow{2}{*}{ Follow-Up Time } & \multicolumn{3}{|c|}{ Post COVID-19 Participants } & \multirow{2}{*}{$\begin{array}{c}\begin{array}{c}\text { Disease Severity at } \\
\text { Baseline }\end{array} \\
\text { Severe Cases } \\
n(\%)\end{array}$} \\
\hline & & & & $\mathbf{N}$ & $\begin{array}{c}\text { Age } \\
\text { (Years) }\end{array}$ & $\begin{array}{l}\text { Male } \\
(\%)\end{array}$ & \\
\hline $\begin{array}{l}\text { Abdallah, S. } \\
2021[46]\end{array}$ & Canada & Cross-sectional & $\begin{array}{l}119.9 \text { (SD 16.2) days after positive COVID-19 test } \\
\text { for hospitalized patients and 129.8 (SD 16.5) for } \\
\text { non-hospitalized patients }\end{array}$ & 63 & $\begin{array}{l}59.1 \text { (SD 13.5) for hospitalized } \\
42.4 \text { (SD 12.9) days non-hospitalized }\end{array}$ & $57 \%$ & $\begin{array}{c}25(40 \%) \\
\text { hospitalized }\end{array}$ \\
\hline $\begin{array}{l}\text { Anastasio, F. } \\
2021 \text { [47] }\end{array}$ & Italy & Cross-sectional & 135 (IQR 102-175) days after symptoms onset & 379 & 56.0 (IQR 49-63) & $46 \%$ & $\begin{array}{l}222(58.6 \%) \\
\text { pneumonia }\end{array}$ \\
\hline $\begin{array}{c}\text { Baricich, A. } \\
2021 \text { [48] }\end{array}$ & Italy & Cross-sectional & 124 (SD 17.5) days after hospital discharge & 204 & 57.9 (SD 12.8) & $60 \%$ & $\begin{array}{c}27(13 \%) \\
\text { ICU }\end{array}$ \\
\hline $\begin{array}{l}\text { Bellan, M. } \\
2021[27]\end{array}$ & Italy & Prospective & 4 months after hospital discharge & 238 & $61.0(\mathrm{IQR}$ 50-71) & $60 \%$ & $\begin{array}{l}28(12 \%) \\
\text { ICU }\end{array}$ \\
\hline $\begin{array}{c}\text { Cao, J. } \\
2021[43]\end{array}$ & China & Prospective & 3 months after hospital discharge & 61 & 43.5 (SD 15.9) & $54 \%$ & $\begin{array}{l}57(94 \%) \\
\text { pneumonia } \\
\text { severe pneumonia } \\
\text { critical illness }\end{array}$ \\
\hline $\begin{array}{l}\text { Guler, S. } \\
2021[34]\end{array}$ & Switzerland & Prospective & 128 (IQR 108-144) days from initial symptoms & 113 & $\begin{array}{c}60.3 \text { (SD 12) Severe/critical COVID } \\
52.9 \text { (SD 11) Mild/moderate COVID-19 }\end{array}$ & $59 \%$ & $\begin{array}{l}66(58.4 \%) \\
\text { severe/ } \\
\text { critical } \\
\text { disease }\end{array}$ \\
\hline $\begin{array}{l}\text { Han, X. } \\
2021[28]\end{array}$ & China & Prospective & 175 (SD 20) days after symptoms onset & 114 & 54.0 (SD 12) & $70 \%$ & $\begin{array}{l}114(100 \%) \\
\text { severe } \\
\text { disease }\end{array}$ \\
\hline $\begin{array}{l}\text { Huang, Ch. } \\
2020[29]\end{array}$ & China & Ambi-directional & 186 (175-199) days after symptom onset & 1733 & 57.0 (IQR 47-65) & $52 \%$ & $\begin{array}{c}1294(75 \%) \\
\text { required } \\
\text { supplemental oxygen } \\
(n=1172) \text { or HFNC, } \\
\text { NIV, or IMV }(n=122)\end{array}$ \\
\hline $\begin{array}{c}\text { Jacobson, K. } \\
2021 \text { [49] }\end{array}$ & USA & Cross-sectional & 119.3 (SD 33) days after COVID-19 diagnosis & 118 & 43.3 (SD 14.4) & $53 \%$ & $\begin{array}{c}22(18.6 \%) \\
\text { hospitalized }\end{array}$ \\
\hline
\end{tabular}


Table 1. Cont.

\begin{tabular}{|c|c|c|c|c|c|c|c|}
\hline \multirow{2}{*}{$\begin{array}{l}\text { Author } \\
\text { Year }\end{array}$} & \multirow{2}{*}{ Country } & \multirow{2}{*}{ Study Design } & \multirow{2}{*}{ Follow-Up Time } & \multicolumn{2}{|c|}{ Post COVID-19 Participants } & \multicolumn{2}{|r|}{$\begin{array}{c}\text { Disease Severity at } \\
\text { Baseline }\end{array}$} \\
\hline & & & & $\mathbf{N}$ & $\begin{array}{l}\text { Age } \\
\text { (Years) }\end{array}$ & $\begin{array}{l}\text { Male } \\
(\%)\end{array}$ & $\begin{array}{c}\text { Severe Cases } \\
n(\%)\end{array}$ \\
\hline $\begin{array}{l}\text { Lerum, T. } \\
2020[30]\end{array}$ & Norway & Prospective & 83 (73-90) days after hospital admission & 103 & 59.0 (IQR 49-72) & $52 \%$ & $\begin{array}{l}15(14.6 \%) \\
\text { ICU }\end{array}$ \\
\hline $\begin{array}{l}\text { Liang, L. } \\
2020[31]\end{array}$ & China & Prospective & 3 months after hospital discharge & 76 & $41.3(\mathrm{SD} 13.8)$ & $28 \%$ & $\begin{array}{c}7(9.2 \%) \\
\text { ICU }\end{array}$ \\
\hline $\begin{array}{l}\text { Morin, L. } \\
2021 \text { [36] }\end{array}$ & France & Prospective & 4 months after hospital or ICU discharge & 478 & 60.9 (SD 16.1) & $58 \%$ & $\begin{array}{l}142(30 \%) \\
\text { ICU }\end{array}$ \\
\hline $\begin{array}{l}\text { * Shah, A. } \\
2020 \text { [37] }\end{array}$ & Canada & Prospective & 11.7 weeks after symptoms onset & 60 & $67.0(\mathrm{IQR}$ 54-74) & $68 \%$ & $\begin{array}{c}46(76.7 \%) \\
\text { required } \\
\text { supplemental oxygen }\end{array}$ \\
\hline $\begin{array}{l}\text { Sonnweber, T. } \\
2020 \text { [32] }\end{array}$ & Austria & Prospective & $\begin{array}{l}103 \text { (SD 21) days after diagnosis for second visit } \\
\text { (100 days after onset) }\end{array}$ & 145 & $57.0(14)$ & $55 \%$ & $\begin{array}{c}109(75 \%) \\
\text { hospitalized }\end{array}$ \\
\hline $\begin{array}{l}\text { Sykes, D. } \\
2021 \text { [50] }\end{array}$ & UK & Cross-sectional & 113 days (46-167) days post discharge & 134 & 59.6 (SD 14.0) & $66 \%$ & $\begin{array}{l}27(20.1 \%) \\
\text { ICU }\end{array}$ \\
\hline $\begin{array}{l}\text { Tabatabaei, S. } \\
2020[33]\end{array}$ & Iran & Retrospective & 91 (SD 15.5) days after initial CT & 52 & $50.2(\mathrm{SD} 13.1)$ & $62 \%$ & NR \\
\hline $\begin{array}{l}\text { van den Borst, B. } \\
2020[45]\end{array}$ & $\begin{array}{l}\text { the Nether- } \\
\text { lands }\end{array}$ & Prospective & 13.0 (SD 2.2) weeks after symptoms onset & 124 & 59.0 (SD 14) & $60 \%$ & $\begin{array}{l}46(37 \%) \\
\text { severe/ } \\
\text { critical } \\
\text { disease }\end{array}$ \\
\hline $\begin{array}{l}\text { Walle-Hansen, M. } \\
\text { 2021 [38] }\end{array}$ & Norway & Prospective & 186 days after discharge & 106 & 74.3 (range 60-96) & $57 \%$ & $\begin{array}{c}27(25.4 \%) \\
\text { ICU or } \\
\text { intermediary ward }\end{array}$ \\
\hline $\begin{array}{l}{ }^{*} \text { Wong, A. } \\
2020[35]\end{array}$ & Canada & Prospective & 13 (IQR 11-14) weeks after symptoms onset & 78 & 62.0 (SD 16) & $64 \%$ & NR \\
\hline
\end{tabular}


Table 1. Cont.

\begin{tabular}{|c|c|c|c|c|c|c|c|}
\hline \multirow{2}{*}{$\begin{array}{l}\text { Author } \\
\text { Year }\end{array}$} & \multirow{2}{*}{ Country } & \multirow{2}{*}{ Study Design } & \multirow{2}{*}{ Follow-Up Time } & \multicolumn{2}{|c|}{ Post COVID-19 Participants } & \multirow{2}{*}{\multicolumn{2}{|c|}{$\begin{array}{lc} & \begin{array}{c}\text { Disease Severity at } \\
\text { Baseline }^{¥}\end{array} \\
\begin{array}{l}\text { Male } \\
(\%)\end{array} & \text { Severe Cases } \\
& n(\%)\end{array}$}} \\
\hline & & & & $\mathbf{N}$ & $\begin{array}{c}\text { Age } \\
\text { (Years) }\end{array}$ & & \\
\hline $\begin{array}{l}\text { Wu, Q. } \\
2021[41]\end{array}$ & China & Prospective & 6 months after discharge & 54 & 47.0 (IQR 36-57) & $59 \%$ & $\begin{array}{l}23(42.5 \%) \\
\text { severe } \\
\text { disease }\end{array}$ \\
\hline $\begin{array}{l}\text { Xiong, Q. } \\
2021[42]\end{array}$ & China & Prospective & 97 (95-102) days after hospital discharge & 538 & 52.0 (SD 41-62) & $46 \%$ & $\begin{array}{c}207(38.5 \%) \\
\text { severe/ } \\
\text { critical } \\
\text { disease }\end{array}$ \\
\hline $\begin{array}{l}\text { Zhao, Y. } \\
2020[40]\end{array}$ & China & Retrospective & $\begin{array}{c}3 \text { months after symptom onset (64-93 days after } \\
\text { discharged from hospitals) }\end{array}$ & 55 & 47.7 (SD 15.49) & $58 \%$ & $\begin{array}{l}4(7.3 \%) \\
\text { severe } \\
\text { disease }\end{array}$ \\
\hline
\end{tabular}

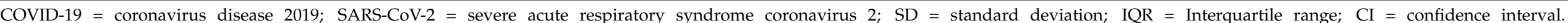

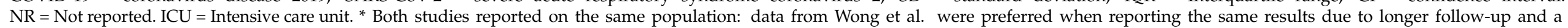

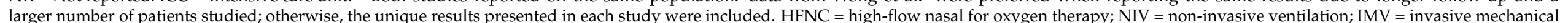
ventilation. $¥$ As described in the articles. 
Table 2. Prevalence of persistent COVID-19 effects at 3 to 6 moths follow-up.

\begin{tabular}{|c|c|c|c|c|c|c|}
\hline Outcome & No. of Studies & $\begin{array}{l}\text { No. of Patients } \\
\text { Analyzed }\end{array}$ & $\begin{array}{c}\text { Mean (SD) } \\
\text { Follow-Up Time } \\
\text { (Months) }\end{array}$ & Pooled Prevalence $(95 \%$ CI $)$ & $\mathbf{I}^{2} \%$ & $p$-Value \\
\hline \multicolumn{7}{|c|}{ Chest CT [28-34,36,37,40,41,43,45] } \\
\hline Chest $\mathrm{CT}$ abnormalities & 10 & 987 & $3.9(1.4)$ & $59 \%(44-73 \%)$ & 96 & $<0.001$ \\
\hline GGO & 13 & 1313 & $3.9(1.3)$ & $39 \%(26-52 \%)$ & 97 & $<0.001$ \\
\hline $\begin{array}{l}\text { Interstitial thickening or interlobular septal } \\
\text { thickening }\end{array}$ & 7 & 885 & $3.8(1.2)$ & $33 \%(13-52 \%)$ & 98 & $<0.001$ \\
\hline Parenchymal band or fibrous stripe & 6 & 815 & $3.8(1.2)$ & $31 \%(17-44 \%)$ & 95 & $<0.001$ \\
\hline Bronchovascular bundle distortion or bronchiectasis & 5 & 437 & $4.5(1.3)$ & $26 \%(9-43 \%)$ & 97 & $<0.001$ \\
\hline Thickening or adjacent pleura & 4 & 573 & $5.5(0.8)$ & $11 \%(2-20 \%)$ & 94 & $<0.001$ \\
\hline Consolidation & 4 & 652 & $4.9(1.3)$ & $6 \%(2-11 \%)$ & 89 & $<0.001$ \\
\hline Crazy paving & 1 & 55 & 3.0 & $5 \%(1-11 \%)$ & NA & NA \\
\hline \multicolumn{7}{|c|}{ Pulmonary function $[27-32,36,37,40,41,43,45,46]$} \\
\hline Pulmonary function abnormalities & 6 & 439 & $3.5(1.2)$ & $39 \%(24-55 \%)$ & 94 & $<0.001$ \\
\hline Diffusion pattern & 12 & 1490 & $4.0(1.3)$ & $31 \%(24-38 \%)$ & 89 & $<0.001$ \\
\hline Restrictive Pattern & 8 & 921 & $3.8(1.5)$ & $12 \%(8-17 \%)$ & 82 & $<0.001$ \\
\hline Obstructive pattern & 7 & 858 & $3.6(1.3)$ & $8 \%(6-9 \%)$ & 7 & $<0.001$ \\
\hline \multicolumn{7}{|c|}{ Fatigue $[29,31,32,35,36,39-41,43-47,49,50]$ and respiratory symptoms $[27-33,35,36,40-44,46,47,49,50]$} \\
\hline Fatigue & 15 & 4118 & $4.0(1.3)$ & $38 \%(27-49 \%)$ & 98 & $<0.001$ \\
\hline Dyspnea & 16 & 3526 & $4.0(1.1)$ & $32 \%(24-40 \%)$ & 98 & $<0.001$ \\
\hline Chest pain/Tightness & 10 & 3728 & $3.9(0.9)$ & $16 \%(12-21 \%)$ & 94 & $<0.001$ \\
\hline Cough & 14 & 2539 & $3.8(0.9)$ & $13 \%(9-17 \%)$ & 94 & $<0.001$ \\
\hline Sputum & 4 & 783 & $3.8(1.4)$ & $12 \%(3-21 \%)$ & 94 & $<0.001$ \\
\hline Sore Throat & 6 & 2554 & $4.4(1.3)$ & $4 \%(2-7 \%)$ & 66 & 0.02 \\
\hline
\end{tabular}


Table 2. Cont.

\begin{tabular}{|c|c|c|c|c|c|c|}
\hline Outcome & No. of Studies & $\begin{array}{l}\text { No. of Patients } \\
\text { Analyzed }\end{array}$ & $\begin{array}{c}\text { Mean (SD) } \\
\text { Follow-Up Time } \\
\text { (Months) }\end{array}$ & Pooled Prevalence $(95 \% \mathrm{CI})$ & $\mathrm{I}^{2} \%$ & $p$-Value \\
\hline \multicolumn{7}{|c|}{ Functional capacity $[27,29,45,48]$, Health-related quality of live (HRQoL) $[35,38,39,45]$, and return to work/work impairment $[31,40,44,49]$} \\
\hline * Decreased functional capacity & 5 & 2364 & $4.2(1.3)$ & $36 \%(22-49 \%)$ & 97 & $<0.001$ \\
\hline Decreased HRQoL & 4 & 474 & $4.4(1.6)$ & $52 \%(33-71 \%)$ & 94 & $<0.001$ \\
\hline$¥$ Return to work/No work impairment & 4 & 259 & $3.4(0.4)$ & $80 \%$ & NA & NA \\
\hline
\end{tabular}

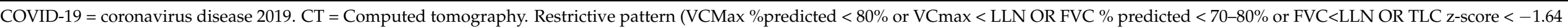

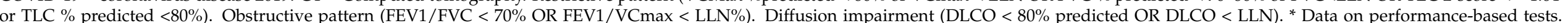

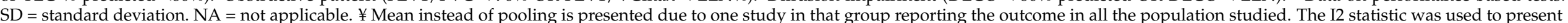

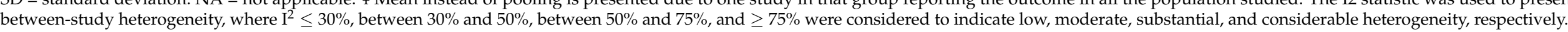




\subsection{Lung Function}

Thirteen articles explored the effect of COVID-19 on lung function at follow-up $[27-32,36,37,40,41,43,45,46]$ (Supplementary Table S3 and Figure S2). Abnormal pulmonary function tests (PFT) were identified in 39\% (95\% CI 24-55, $\mathrm{I}^{2}=94 \%$ ) of the patients (Table 2). Impaired diffusion lung capacity was the most common finding in 31\% (95\% CI 24-38, $\left.\mathrm{I}^{2}=89 \%\right)$ of the patients assessed, followed by restrictive pattern in $12 \%$ (95\% CI 8-17, $\left.\mathrm{I}^{2}=82 \%\right)$, and obstructive pattern in $8 \%\left(95 \%\right.$ CI 6-9, $\left.\mathrm{I}^{2}=7 \%\right)$. DLCO was lower in hospitalized vs. non-hospitalized patients, however, abnormal values were observed in both groups [46]. Mean DLCO was significantly lower in moderate-to-critical disease patients compared to referred mild disease patients who had normal mean DLCO [34,45]. No impact of disease severity in lung function are reported in other studies $[30,41]$.

\subsection{Fatigue and Respiratory Symptoms}

Fifteen studies explored persistent post COVID-19 fatigue [29,31,32,35,36,39-41,43-47, $49,50]$ and eighteen studies [27-33,35,36,40-44,46,47,49,50] report on one or more persistent respiratory symptoms at follow-up (Supplementary Table S4 and Figure S3). Pooled analysis indicates that fatigue was reported by $38 \%$ of the patients $\left(95 \% \mathrm{CI} 27-49, \mathrm{I}^{2}=98 \%\right)$ (Table 2), dyspnea by $32 \%\left(95 \%\right.$ CI $\left.24-40, \mathrm{I}^{2}=9 \%\right)$, chest pain or tightness by $16 \%(95 \% \mathrm{CI}$ $\left.12-21, \mathrm{I}^{2}=94 \%\right)$, cough by $13 \%,\left(95 \%\right.$ CI $\left.9-17, \mathrm{I}^{2}=94 \%\right)$, sputum by $12 \%\left(95 \%\right.$ CI $3-21, \mathrm{I}^{2}=$ $94 \%)$, and sore throat by $4 \%\left(95 \%\right.$ CI $\left.2-7, \mathrm{I}^{2}=66 \%\right)$. One study reports a higher prevalence of fatigue in patients with severe COVID-19 [29], and another found a lower prevalence of dyspnea (mMRC) in patients with moderate-to-critical illness compared with patients with mild disease [45]. However, disease severity is not significantly associated with the prevalence of fatigue or respiratory symptoms at follow-up in other studies $[30,35,41,42,44]$.

\subsection{Functional Capacity, HRQoL, and Return to Work/No Work Impairment}

Decreased functional capacity was identified in an average of 36\% (95\% CI 22-49, $\mathrm{I}^{2}=97 \%$ ) of the patients at a mean of 4.2 (SD 1.3) months follow-up using data from five studies which presented a proportion of decreased functionality assessed with the work productivity and impairment questionnaire (WPAI) [49] and with performance-based tests (Table 2) including the short physical performance battery (SPPB) [27,48], the one minute sit to stand test (1-MSTST) [48], the 2 min walking test [27,48], and the 6 minute walking test (6MWT) [29,45] (Supplementary Table S5 and Figure S4). Cao et al. also stated that performance in the 6MWT was significantly lower in post COVID-19 patients than in health controls but did not report the proportion of patients under the threshold [43]. Lower performance in the 6MWT was reported in patients with severe/critical COVID19 compared to patients with mild/moderate disease at baseline [29,34,47,49], however, another study reports no difference between groups [30].

Four studies report decreased HRQoL in an average of 52\% $\left(95 \% 33-71, \mathrm{I}^{2}=94 \%\right)$ of the participants at a mean of 4.5 (SD 1.7) months follow-up (Table 2) assessed with the Nijmegen Clinical Screening Instrument (NCSI) [45], a sliding scale ranging from 0 to 100 (best) [39], and using the 5 level EuroQoL 5 Dimensions (EQ-5D-5L) [35, 38] (Supplementary Table S5). Patients who experienced severe COVID-19 had larger declines in HRQoL (EQ-5D5L) $[29,30,38]$. Conversely, patients with mild disease reported a significatively worse health status on most subscales of the SF-36, and on the subdomains of the NCSI, compared with moderate-to-critical disease patients [45]. No significant correlation between HRQoL or level of physical activity and disease severity at baseline is reported in other studies [44,47].

Results from four studies indicate that on average $80 \%$ of patients had returned to their previous work or reported no work impairments at a mean of 3.4 (SD 0.4) months followup $[31,40,44,49]$ (Supplementary Table S5). A large percentage of ward hospitalized patients (75.6\%) returned to work compared to ICU patients $(46.7 \%)$, and more work impairment was reported in hospitalized patients (58.3\%) compared to those not hospitalized (35.0\%), however, the differences were not statistically significant [44,49]. Pooled analysis was not completed as one study identified that all the participants had returned to work. 


\section{Discussion}

Results of this literature review and meta-analysis indicate that post-COVID-19 patients may experience persistent chest $\mathrm{CT}$ abnormalities, decreased lung function, persistent fatigue, and respiratory symptoms, decreased functional capacity, and decreased quality of life up to 6 months after symptom onset or hospital discharge. On average, 8 out of 10 patients had returned to work or reported no work impairment at around 3 months of follow-up.

Prevalence of chest $\mathrm{CT}$ abnormalities and decreased pulmonary function post-COVID19 aligned with evidence from previous studies, which report abnormal outcomes in patients with viral pneumonias up to 64 months after illness onset [51,52]. Persistent lung abnormalities are significantly associated with disease severity at baseline in various studies $[30,33,34,41,45]$. However, high prevalence of chest CT abnormalities was not well reflected on PFT and only weak to moderate correlations were found [31,32]. Presence of GGO, commonly identified at follow-up [28-34,36,37,40,41,43,45], can be a manifestation of a wide variety of clinical features including focal interstitial fibrosis, and inflammation [53]. Residual pulmonary parenchymal abnormalities were correlated with lower DLCO [45], and pulmonary function abnormalities occurred frequently in patients with fibrotic-like changes [28]. Impaired DLCO reported in the PFT [27-32,36,37,40,41,45] can be present in interstitial lung diseases or pulmonary fibrosis, which are clinical conditions where the surface area of the alveolar membrane is reduced [54]. DLCO values represent the ability of the lung to transfer gas from the inhaled air into the blood stream and can be used as a marker of the extent of lung damage [55]. Impaired DLCO may be attributed to abnormalities in the distal airways (such as constrictive bronchiolitis with air trapping and secondary reflex vasoconstriction), or to primary vascular disease (that may induce secondary airway disease) [34]. Abnormal DLCO and GGO on chest CT were associated with duration of oxygen supplementation [37].

High prevalence of cough, dyspnea, and fatigue were reported in post COVID-19 patients. Cough and dyspnea can be indicators of impaired lung function [54] and may also affect the execution of an adequate PFT [56]. Dyspnea scores were found to correlate with PFT measurements in COVID-19 patients [31]. Presence of dyspnea is attributed to abnormalities on lung CT scan in 44 of 78 patients (45\%), including fibrotic lesions in 18 of 78 cases $(23 \%)$ and to hyperventilation functional breathing test confirmed dysfunctional breathing in 14 of 78 patients (17\%) [36]. Although some evidence supports the theory that breathing problems in COVID-19 patients may be due to fibrotic changes in the lung, it is also suggested that post-viral autonomic dysfunction may contribute to the cause of this and other persistent symptoms [57]. Fatigue, the most prevalent symptom reported by post COVID-19 patients, was identified in one third of the patients and could likely persist for a longer time. A previous study reports that $40 \%$ of SARS survivors had chronic fatigue for a mean of 41 months after infection [58]. Post-COVID-19 fatigue is not associated with disease severity in the acute phase or with abnormal chest CT abnormalities [22]. Fatigue was reported together with muscle weakness in one of the studies [42], and with physical decline in another [29]. It is possible that fatigue may have been caused by muscle weakness, respiratory symptoms, and general deconditioning. It has also been suggested that the coronavirus can trigger post-viral fatigue syndromes [59]. More studies will be needed to clarify the cause of fatigue in post-COVID-19 patients and the best way to treat it.

Decreased functional capacity was found in one third of post COVID-19 patients at follow-up. CT abnormalities are not associated with functional performance [41], however, residual signs suggesting pulmonary fibrosis are associated with exercise-induced desaturation [45]. Sixteen percent of patients desaturated upon the 6MWT, desaturators had lower DLCO than non-desaturators [45]. Persistent symptoms, decreased pulmonary function, muscle weakness and physical deconditioning may have contributed to post COVID-19 decreased function and perception of worsened HRQoL. Despite the higher prevalence of multiple post-COVID-19 effects, a large percentage of the patients had returned to work 3 to 4 months after discharge $[31,40,44]$. Importantly, all studies that reported on this 
outcome used small sample sizes (ranging from 55 to 76 patients). Furthermore, in the studies that found that all or almost all patients had returned to work, the participants were relatively young (mean age 41.3 SD13.8 and 47.7 SD 15.4 years) and only a small percentage of them had a serious illness that required ICU care (Table 1) [31,40]. Although in Garrigues et al. the group studied was older (mean 63.2 SD 15.7), this outcome was reported only in $46.7 \%(56 / 120)$ of the participants, probably younger patients still active in the workforce. Therefore, a potential explanation for the lower return to work (68\%) could be a higher percentage of patients with severe disease at baseline, since $20 \%$ of participants were in the ICU [44]. It is also possible that the socio-economic context and available resources may have an impact on the return-to-work process. In general, more studies with larger sample sizes are needed to determine the potential long-term impact of COVID-19 on patients ability to return to work, as well as the possible causes and ways to mitigate them.

Patients with severe COVID-19 disease had higher prevalence of chest CT abnormalities $[30,33,34,41,45]$. However, inconclusive results were found regarding disease severity and persistent fatigue, respiratory symptoms, pulmonary function, functional capacity, and HRQoL. Evidence suggests that severity of disease during the acute period of infection may have an impact on persistent COVID-19 outcomes. However, only a few studies report the outcomes in relation with disease severity and no direct association between disease severity and post COVID-19 outcomes was observed. Persistent symptoms are also reported in patients with non-severe disease.

\subsection{Implications and Considerations}

This study helps fill a significant knowledge gap about the lingering effects of COVID19 on patient outcomes between 3 to 6 months after infection, which is very important considering the large number of COVID-19 patients who will potentially need help managing the impact of the disease beyond the infectious period. Outcomes of this study may help to understand the prevalence of persistent post-COVID-19 effects and their potential causes, which may guide the development of rehabilitation programs tailored to the needs of COVID-19 patients. From a public health perspective, the results of this study may help inform future health care planning and resources allocation.

\subsection{Study Limitations}

Some factors should be considered when interpreting these findings. First, there is no data available on outcomes studied before the patients contracted COVID-19, therefore, it is unclear whether the abnormalities may have existed before the diagnosis of COVID-19. Second, it is possible that the presence of pre-existent comorbidities may have affected the results of the studies. However, Wong et al. [35] report that there were no differences in reported outcomes between patients with and without pre-existing major comorbidities, suggesting that many of the identified deficits are likely ongoing consequences of COVID-19. Third, there is heterogeneity across the studies with respect to the selection of participants, the assessment of outcomes, and the definition of the follow-up period in almost all the studies which may influence the generalizability of the results of this study. Despite these limitations, the authors believe that the results of this study contribute to filling a significant knowledge gap about persistent COVID-19 symptoms between 3 and 6 months after acute infection.

\section{Conclusions}

The results indicate that post-COVID-19 patients may experience persistent respiratory symptoms, fatigue, decreased functional capacity, and decreased quality of life up to 6 months after infection and, on average, around 8 out of 10 of the patients had returned to work at around 3 months follow-up. Outcomes suggest that, in addition to disease severity and lung injury, other potential causes of persistent symptoms, such as postviral autonomic dysfunction and fatigue syndrome should be assessed and considered when planning post-COVID-19 rehabilitation interventions. This study may guide the 
development of rehabilitation programs tailored to the needs of COVID-19 patients and inform future health care planning and resource allocation. Further studies are needed to establish the extent to which post-COVID-19 effects continue beyond 6 months, how they interact with each other, and to clarify their causes and their effective management.

Supplementary Materials: The following are available online at https://www.mdpi.com/article/10 .3390/biomedicines9080900/s1, Table S1: Quality Assessment Tool for Observational Cohort and Cross-Sectional Studies, Table S2: Prevalence of chest CT abnormalities $\geq 3$ months post COVID-19, Table S3: Prevalence of pulmonary function abnormalities $\geq 3$ months post COVID-19, Table S4: Prevalence of fatigue and respiratory symptoms $\geq 3$ months post COVID-19, Table S5: Prevalence of decreases functional capacity and health-related quality of life, and return to work $\geq 3$ post COVID19, Figure S1: Forest plots for follow-up chest CT, Figure S2: Forest plots for follow-up pulmonary function, Figure S3: Forest plots for follow-up respiratory symptoms, Figure S4: Functional capacity and Health-related quality of life.

Author Contributions: Study concept and design: D.C.S.-R.; acquisition, analysis, or interpretation of data: D.C.S.-R., K.N., Z.Y. and R.T.-C., D.C.S.-R. and R.T.-C.; drafting of the manuscript: D.C.S.-R. and R.T.-C.; critical revision of the manuscript for important intellectual content: D.C.S.-R. and R.T.-C.; statistical analysis: D.C.S.-R.; administrative, technical, or material support: K.N. and Z.Y. All authors have read and agreed to the published version of the manuscript.

Funding: Z.Y. received funding from the 2021 Globalink Research Internship Program while participating in this study.

Institutional Review Board Statement: Not applicable.

Informed Consent Statement: Not applicable.

Conflicts of Interest: The authors declare no conflict of interest.

\section{References}

1. John Hopkins University of Medicine Coronavirus Resource Center. Available online: https://coronavirus.jhu.edu/ (accessed on 21 July 2021).

2. Shi, H.; Han, X.; Jiang, N.; Cao, Y.; Alwalid, O.; Gu, J.; Fan, Y.; Zheng, C. Radiological findings from 81 patients with COVID-19 pneumonia in Wuhan, China: A descriptive study. Lancet Infect. Dis. 2020, 20, 425-434. [CrossRef]

3. Wu, Z.; McGoogan, J.M. Characteristics of and important lessons from the coronavirus disease 2019 (COVID-19) outbreak in China: Summary of a report of 72,314 cases from the Chinese Center for Disease Control and Prevention. JAMA 2020, 323, 1239-1242. [CrossRef]

4. Nöbauer-Huhmann, I.-M.; Eibenberger, K.; Schaefer-Prokop, C.; Steltzer, H.; Schlick, W.; Strasser, K.; Fridrich, P.; Herold, C.J. Changes in lung parenchyma after acute respiratory distress syndrome (ARDS): Assessment with high-resolution computed tomography. Eur. Radiol. 2001, 11, 2436-2443. [CrossRef]

5. Xie, L.; Liu, Y.; Xiao, Y.; Tian, Q.; Fan, B.; Zhao, H.; Chen, W. Follow-up Study on Pulmonary Function and Lung Radiographic Changes in Rehabilitating Severe Acute Respiratory Syndrome Patients After Discharge. Chest 2005, 127, 2119-2124. [CrossRef]

6. Hui, D.S.; Joynt, G.; Wong, K.T.; Gomersall, C.; Li, T.S.; Antonio, G.; Ko, F.W.S.; Chan, M.C.; Chan, D.P.; Tong, M.W.; et al. Impact of severe acute respiratory syndrome (SARS) on pulmonary function, functional capacity and quality of life in a cohort of survivors. Thorax 2005, 60, 401-409. [CrossRef] [PubMed]

7. Ngai, J.C.; Ko, F.W.S.; Ng, S.; To, K.-W.; Tong, M.; Hui, D. The long-term impact of severe acute respiratory syndrome on pulmonary function, exercise capacity and health status. Respirology 2010, 15, 543-550. [CrossRef]

8. Ong, K.-C.; Ng, A.W.-K.; Lee, L.; Kaw, G.; Kwek, S.-K.; Leow, M.K.-S.; Earnest, A. 1-Year Pulmonary Function and Health Status in Survivors of Severe Acute Respiratory Syndrome. Chest 2005, 128, 1393-1400. [CrossRef]

9. Liu, W.; Peng, L.; Liu, H.; Hua, S. Pulmonary Function and Clinical Manifestations of Patients Infected with Mild Influenza A Virus Subtype H1N1: A One-Year Follow-Up. PLoS ONE 2015, 10, e0133698. [CrossRef]

10. Luyt, C.-E.; Combes, A.; Becquemin, M.-H.; Beigelman-Aubry, C.; Hatem, S.; Brun, A.-L.; Zraik, N.; Carrat, F.; Grenier, P.A.; Richard, J.-C.M.; et al. Long-term Outcomes of Pandemic 2009 Influenza A(H1N1)-Associated Severe ARDS. Chest 2012, 142, 583-592. [CrossRef] [PubMed]

11. Bein, T.; Weber-Carstens, S.; Apfelbacher, C. Long-term outcome after the acute respiratory distress syndrome: Different from general critical illness? Curr. Opin. Crit. Care 2018, 24, 35-40. [CrossRef]

12. Schelling, G.; Stoll, C.; Vogelmeier, C.; Hummel, T.; Behr, J.; Kapfhammer, H.-P.; Rothenhäusler, H.-B.; Haller, M.; Durst, K.; Krauseneck, T.; et al. Pulmonary function and health-related quality of life in a sample of long-term survivors of the acute respiratory distress syndrome. Intensive Care Med. 2000, 26, 1304-1311. [CrossRef] [PubMed] 
13. Wilcox, M.E.; Herridge, M.S. Lung function and quality of life in survivors of the acute respiratory distress syndrome (ARDS). La Presse Médicale 2011, 40, e595-e603. [CrossRef] [PubMed]

14. Mahase, E. COVID-19: What do we know about long covid? BMJ 2020, 370, m2815. [CrossRef]

15. Huang, Y.; Tan, C.; Wu, J.; Chen, M.; Wang, Z.; Luo, L.; Zhou, X.; Liu, X.; Huang, X.; Yuan, S.; et al. Impact of coronavirus disease 2019 on pulmonary function in early convalescence phase. Respir. Res. 2020, 21, 1-10. [CrossRef]

16. Cellai, M.; O'Keefe, J.B. Characterization of Prolonged COVID-19 Symptoms in an Outpatient Telemedicine Clinic. Open Forum Infect. Dis. 2020, 7, ofaa420. [CrossRef]

17. Daher, A.; Balfanz, P.; Cornelissen, C.; Müller, A.; Bergs, I.; Marx, N.; Müller-Wieland, D.; Hartmann, B.; Dreher, M.; Müller, T. Follow up of patients with severe coronavirus disease 2019 (COVID-19): Pulmonary and extrapulmonary disease sequelae. Respir. Med. 2020, 174, 106197. [CrossRef]

18. Jacobs, L.G.; Paleoudis, E.G.; Bari, D.L.-D.; Nyirenda, T.; Friedman, T.; Gupta, A.; Rasouli, L.; Zetkulic, M.; Balani, B.; Ogedegbe, C.; et al. Persistence of symptoms and quality of life at 35 days after hospitalization for COVID-19 infection. PLoS ONE 2020, 15, e0243882. [CrossRef]

19. Halpin, S.J.; McIvor, C.; Whyatt, G.; Adams, A.; Harvey, O.; McLean, L.; Walshaw, C.; Kemp, S.; Corrado, J.; Singh, R.; et al. Postdischarge symptoms and rehabilitation needs in survivors of COVID-19 infection: A cross-sectional evaluation. J. Med. Virol. 2021, 93, 1013-1022. [CrossRef]

20. Pizarro-Pennarolli, C.; Sánchez-Rojas, C.; Torres-Castro, R.; Vera-Uribe, R.; Sanchez-Ramirez, D.C.; Vasconcello-Castillo, L.; Solís-Navarro, L.; Rivera-Lillo, G. Assessment of activities of daily living in patients post COVID-19: A systematic review. PeerJ 2021, 9, e11026. [CrossRef]

21. Carfi, A.; Bernabei, R.; Landi, F. Persistent Symptoms in Patients After Acute COVID-19. JAMA 2020, 324, 603-605. [CrossRef]

22. Townsend, L.; Dyer, A.H.; Jones, K.; Dunne, J.; Mooney, A.; Gaffney, F.; O'Connor, L.; Leavy, D.; O’Brien, K.; Dowds, J.; et al. Persistent fatigue following SARS-CoV-2 infection is common and independent of severity of initial infection. PLoS ONE 2020, 15, e0240784. [CrossRef] [PubMed]

23. D'Cruz, R.F.; Waller, M.D.; Perrin, F.; Periselneris, J.; Norton, S.; Smith, L.-J.; Patrick, T.; Walder, D.; Heitmann, A.; Lee, K.; et al. Chest radiography is a poor predictor of respiratory symptoms and functional impairment in survivors of severe COVID-19 pneumonia. ERJ Open Res. 2021, 7. [CrossRef]

24. Moher, D.; Liberati, A.; Tetzlaff, J.; Altman, D.G. Preferred Reporting Items for Systematic Reviews and Meta-Analyses: The PRISMA Statement. Ann. Intern. Med. 2009, 151, 264-269. [CrossRef]

25. National Heart, Lung and Blood Institute. Quality Assessment Tool for Observational Cohort and Cross-Sectional Studies. Available online: https:/ /www.nhlbi.nih.gov/health-topics/study-quality-assessment-tools (accessed on 4 June 2021).

26. Higgins, J.; Thomas, J. Cochrane Handbook for Systematic Reviews of Interventions. Available online: https://training.cochrane. org/handbook/current (accessed on 4 June 2021).

27. Bellan, M.; Soddu, D.; Balbo, P.E.; Baricich, A.; Zeppegno, P.; Avanzi, G.C.; Baldon, G.; Bartolomei, G.; Battaglia, M.; Battistini, S.; et al. Respiratory and Psychophysical Sequelae Among Patients With COVID-19 Four Months After Hospital Discharge. JAMA Netw. Open 2021, 4, e2036142. [CrossRef]

28. Han, X.; Fan, Y.; Alwalid, O.; Li, N.; Jia, X.; Yuan, M.; Li, Y.; Cao, Y.; Gu, J.; Wu, H.; et al. Six-month Follow-up Chest CT Findings after Severe COVID-19 Pneumonia. Radiology 2021, 299, E177-E186. [CrossRef]

29. Huang, C.; Huang, L.; Wang, Y.; Li, X.; Ren, L.; Gu, X.; Kang, L.; Guo, L.; Liu, M.; Zhou, X.; et al. 6-month consequences of COVID-19 in patients discharged from hospital: A cohort study. Lancet 2021, 397, 220-232. [CrossRef]

30. Lerum, T.V.; Aaløkken, T.M.; Brønstad, E.; Aarli, B.; Ikdahl, E.; Lund, K.M.A.; Durheim, M.T.; Rodriguez, J.R.; Meltzer, C.; Tonby, K.; et al. Dyspnoea, lung function and CT findings three months after hospital admission for COVID-19. Eur. Respir. J. 2020, 57, 2003448. [CrossRef]

31. Liang, L.; Yang, B.; Jiang, N.; Fu, W.; He, X.; Zhou, Y.; Ma, W.-L.; Wang, X. Three-Month Follow-Up Study of Survivors of Coronavirus Disease 2019 after Discharge. J. Korean Med. Sci. 2020, 35, e418. [CrossRef]

32. Sonnweber, T.; Sahanic, S.; Pizzini, A.; Luger, A.; Schwabl, C.; Sonnweber, B.; Kurz, K.; Koppelstätter, S.; Haschka, D.; Petzer, V.; et al. Cardiopulmonary recovery after COVID-19-An observational prospective multi-center trial. Eur. Respir J. 2021, 57, 2003481.

33. Tabatabaei, S.M.H.; Rajebi, H.; Moghaddas, F.; Ghasemiadl, M.; Talari, H. Chest CT in COVID-19 pneumonia: What are the findings in mid-term follow-up? Emerg. Radiol. 2020, 27, 711-719. [CrossRef]

34. Guler, S.A.; Ebner, L.; Aubry-Beigelman, C.; Bridevaux, P.-O.; Brutsche, M.; Clarenbach, C.; Garzoni, C.; Geiser, T.K.; Lenoir, A.; Mancinetti, M.; et al. Pulmonary function and radiological features 4 months after COVID-19: First results from the national prospective observational Swiss COVID-19 lung study. Eur. Respir. J. 2021, 57, 2003690. [CrossRef]

35. Wong, A.W.; Shah, A.S.; Johnston, J.C.; Carlsten, C.; Ryerson, C.J. Patient-reported outcome measures after COVID-19: A prospective cohort study. Eur. Respir. J. 2020, 56, 2003276. [CrossRef] [PubMed]

36. The Writing Committee for the COMEBAC Study Group; Morin, L.; Savale, L.; Pham, T.; Colle, R.; Figueiredo, S.; Harrois, A.; Gasnier, M.; Lecoq, A.-L.; Meyrignac, O.; et al. Four-Month Clinical Status of a Cohort of Patients After Hospitalization for COVID-19. JAMA 2021, 325, 1525. [CrossRef]

37. Shah, A.S.; Wong, A.W.; Hague, C.J.; Murphy, D.T.; Johnston, J.C.; Ryerson, C.J.; Carlsten, C. A prospective study of 12-week respiratory outcomes in COVID-19-related hospitalisations. Thorax 2021, 76, 402-404. [CrossRef] 
38. Walle-Hansen, M.M.; Ranhoff, A.H.; Mellingsæter, M.; Wang-Hansen, M.S.; Myrstad, M. Health-related quality of life, functional decline, and long-term mortality in older patients following hospitalisation due to COVID-19. BMC Geriatr. 2021, 21, 1-10. [CrossRef] [PubMed]

39. Logue, J.K.; Franko, N.M.; McCulloch, D.J.; McDonald, D.; Magedson, A.; Wolf, C.R.; Chu, H.Y. Sequelae in Adults at 6 Months After COVID-19 Infection. JAMA Netw. Open 2021, 4, e210830. [CrossRef] [PubMed]

40. Zhao, Y.-M.; Shang, Y.-M.; Song, W.-B.; Li, Q.-Q.; Xie, H.; Xu, Q.-F.; Jia, J.-L.; Li, L.-M.; Mao, H.-L.; Zhou, X.-M.; et al. Follow-up study of the pulmonary function and related physiological characteristics of COVID-19 survivors three months after recovery. EClinicalMedicine 2020, 25, 100463. [CrossRef]

41. Wu, Q.; Zhong, L.; Li, H.; Guo, J.; Li, Y.; Hou, X.; Yang, F.; Xie, Y.; Li, L.; Xing, Z. A Follow-Up Study of Lung Function and Chest Computed Tomography at 6 Months after Discharge in Patients with Coronavirus Disease. Can. Respir. J. 2021, $2021,1-7$. [CrossRef]

42. Xiong, Q.; Xu, M.; Li, J.; Liu, Y.; Zhang, J.; Xu, Y.; Dong, W. Clinical sequelae of COVID-19 survivors in Wuhan, China: A single-centre longitudinal study. Clin. Microbiol. Infect. 2021, 27, 89-95. [CrossRef]

43. Cao, J.; Zheng, X.; Wei, W.; Chu, X.; Chen, X.; Wang, Y.; Liu, Q.; Luo, S.; Weng, J.; Hu, X. Three-month outcomes of recovered COVID-19 patients: Prospective observational study. Ther. Adv. Respir. Dis. 2021, 15. [CrossRef]

44. Garrigues, E.; Janvier, P.; Kherabi, Y.; Le Bot, A.; Hamon, A.; Gouze, H.; Doucet, L.; Berkani, S.; Oliosi, E.; Mallart, E.; et al. Post-discharge persistent symptoms and health-related quality of life after hospitalization for COVID-19. J. Infect. 2020, 81, e4-e6. [CrossRef]

45. van den Borst, B.; Peters, J.B.; Brink, M.; Schoon, Y.; Bleeker-Rovers, C.P.; Schers, H.; van Hees, H.W.H.; van Helvoort, H.; van den Boogard, M.; van der Hoeven, H.; et al. Comprehensive health assessment three months after recovery from acute COVID-19. Clin. Infect. Dis. 2020, ciaa1750. [CrossRef]

46. Abdallah, S.J.; Voduc, N.; Corrales-Medina, V.F.; McGuinty, M.; Pratt, A.; Chopra, A.; Law, A.; Garuba, H.; Thavorn, K.; Reid, R.E.; et al. Symptoms, Pulmonary Function and Functional Capacity Four Months after COVID-19. Ann. Am. Thorac. Soc. 2021. [CrossRef]

47. Anastasio, F.; Barbuto, S.; Scarnecchia, E.; Cosma, P.; Fugagnoli, A.; Rossi, G.; Parravicini, M.; Parravicini, P. Medium-term impact of COVID-19 on pulmonary function, functional capacity and quality of life. Eur. Respir. J. 2021, 2004015. [CrossRef] [PubMed]

48. Baricich, A.; Borg, M.B.; Cuneo, D.; Cadario, E.; Azzolina, D.; Balbo, P.; Bellan, M.; Zeppegno, P.; Pirisi, M.; Cisari, C.; et al. Midterm functional sequelae and implications in rehabilitation after COVIDA cross-sectional study. Eur. J. Phys. Rehabil. Med. 2021, 57, 199-207. [CrossRef] [PubMed]

49. Jacobson, K.B.; Rao, M.; Bonilla, H.; Subramanian, A.; Hack, I.; Madrigal, M.; Singh, U.; Jagannathan, P.; Grant, P. Patients With Uncomplicated Coronavirus Disease 2019 (COVID-19) Have Long-Term Persistent Symptoms and Functional Impairment Similar to Patients with Severe COVID-19: A Cautionary Tale During a Global Pandemic. Clin. Infect. Dis. 2021. [CrossRef] [PubMed]

50. Sykes, D.L.; Holdsworth, L.; Jawad, N.; Gunasekera, P.; Morice, A.H.; Crooks, M.G. Post-COVID-19 Symptom Burden: What is Long-COVID and How Should We Manage It? Lung 2021, 199, 113-119. [CrossRef]

51. Hui, D.S.; Wong, K.T.; Ko, F.W.S.; Tam, L.-S.; Chan, D.P.; Woo, J.; Sung, J.J.Y. The 1-Year Impact of Severe Acute Respiratory Syndrome on Pulmonary Function, Exercise Capacity, and Quality of Life in a Cohort of Survivors. Chest 2005, 128, $2247-2261$. [CrossRef]

52. Wang, Q.; Jiang, H.; Xie, Y.; Zhang, T.; Liu, S.; Wu, S.; Sun, Q.; Song, S.; Wang, W.; Deng, X.; et al. Long-term clinical prognosis of human infections with avian influenza A(H7N9) viruses in China after hospitalization. EClinicalMedicine 2020, $20,100282$. [CrossRef]

53. Park, C.M.; Goo, J.M.; Lee, H.J.; Lee, C.H.; Chun, E.J.; Im, J.-G. Nodular Ground-Glass Opacity at Thin-Section CT: Histologic Correlation and Evaluation of Change at Follow-up. Radiographics 2007, 27, 391-408. [CrossRef]

54. Ranu, H.; Wilde, M.; Madden, B. Pulmonary Function Tests. Ulst. Med. J. 2011, 80, 84-90.

55. Ozeki, N.; Kawaguchi, K.; Fukui, T.; Fukumoto, K.; Nakamura, S.; Hakiri, S.; Kato, T.; Hirakawa, A.; Okasaka, T.; Yokoi, $\mathrm{K}$. The diffusing capacity of the lung for carbon monoxide is associated with the histopathological aggressiveness of lung adenocarcinoma. Eur. J. Cardio-Thoracic Surg. 2017, 52, 969-974. [CrossRef] [PubMed]

56. Lundbäck, B.; Stjernberg, N.; Nyström, L.; Forsberg, B.; Lindström, M.; Lundbäck, K.; Jonsson, E.; Rosenhall, L. Epidemiology of respiratory symptoms, lung function and important determinants. Report from the Obstructive Lung Disease in Northern Sweden Project. Tuber. Lung Dis. 1994, 75, 116-126. [CrossRef]

57. Dani, M.; Dirksen, A.; Taraborrelli, P.; Torocastro, M.; Panagopoulos, D.; Sutton, R.; Lim, P.B. Autonomic dysfunction in 'long COVID': Rationale, physiology and management strategies. Clin. Med. 2021, 21, e63-e67. [CrossRef]

58. Lam, M.H.; Wing, Y.K.; Yu, M.W.; Leung, C.M.; Ma, R.C.; Kong, A.P.; So, W.Y.; Fong, S.Y.-Y.; Lam, S.-P. Mental morbidities and chronic fatigue in severe acute respiratory syndrome survivors: Long-term follow-up. Arch. Intern. Med. 2009, 169, $2142-2147$. [CrossRef]

59. Wilson, C. Concern coronavirus may trigger post-viral fatigue syndromes. New Sci. 2020, 246, 10-11. [CrossRef] 